PROCEEDINGS OF THE

AMERICAN MATHEMATICAL SOCIETY

Volume 126, Number 10, October 1998, Pages 2933-2943

S $0002-9939(98) 04437-2$

\title{
DISTRIBUTIONS SUPPORTED IN A HYPERSURFACE AND LOCAL $h^{p}$
}

\author{
GALIA DAFNI \\ (Communicated by Christopher D. Sogge)
}

\begin{abstract}
We give a necessary condition for a distribution with compact support in a hypersurface to be in the local Hardy space $h^{p}\left(\mathbf{R}^{n}\right)$. We apply this condition to prove a result distinguishing two types of Hardy spaces of distributions on a smooth domain $\Omega \subset \mathbf{R}^{n}$.
\end{abstract}

\section{INTRODUCTION}

The aim of this paper is to solve a problem which arose in the study of various local Hardy spaces of distributions on a smooth bounded domain $\Omega \subset \mathbf{R}^{n}$. In [CKS], Chang, Krantz and Stein defined the spaces $h_{r}^{p}(\Omega)$ and $h_{z}^{p}(\Omega), 0<p \leq 1$, (related to the Hardy spaces defined by Miyachi [M] and Jonsson, Sjögren, and Wallin [JSW]) and proceeded to prove regularity results for the Dirichlet and Neumann problems in terms of these spaces. Subsequent work by Chang, Stein and the author ([CDS]) revealed that for certain values of $p$, namely $p \neq \frac{n}{n+k}$, where $k=0,1,2, \ldots$, these two spaces can be identified. However, it was proved that in the one-dimensional case $n=1$, the spaces are not equivalent for the values $p=1, \frac{1}{2}, \frac{1}{3}, \ldots$ Thus it remained to determine whether the two spaces were different for the "critical" values of $p$ for $n>1$.

The space $h_{r}^{p}(\Omega)$ consists of those distributions on $\Omega$ which are the restriction to $\Omega$ of distributions in $h^{p}\left(\mathbf{R}^{n}\right)$, the local Hardy spaces defined by Goldberg (see [G].) The space $h_{z}^{p}(\Omega)$ is a subspace of $h_{r}^{p}(\Omega)$, consisting of those distributions on $\Omega$ which are the restriction to $\Omega$ of distributions in $h^{p}\left(\mathbf{R}^{n}\right)$ which also vanish outside $\bar{\Omega}$. Note that two such extensions of the same element of $h_{z}^{p}(\Omega)$ differ by a distribution in $h^{p}\left(\mathbf{R}^{n}\right)$ which is supported on $\partial \Omega$. Thus in order to understand $h_{z}^{p}(\Omega)$, it is important to understand the nature of distributions in $h^{p}\left(\mathbf{R}^{n}\right)$ which are supported in a smooth hypersurface, or (by a choice of local coordinates) in a hyperplane.

Some simple sufficient conditions can be stated for a distribution with compact support in a hyperplane to be in $h^{p}\left(\mathbf{R}^{n}\right)$ (see for example [S] 5.18 , which implies that $f \in h^{p}\left(\mathbf{R}^{n}\right)$ if its order is strictly less than $\left.\frac{1}{p}-n\right)$. Here we prove a necessary condition, namely that the order of the distribution "in the normal direction" must be strictly less than $n\left(\frac{1}{p}-1\right)$ (Theorem 1 ). We do this by means of a lower bound on the integral of the maximal function of such a distribution on planes parallel to

Received by the editors February 28, 1997.

1991 Mathematics Subject Classification. Primary 42B30, 46 F05.

Key words and phrases. $h^{p}$ spaces, distributions. 
the hyperplane (Lemma 1). It is this lemma that allows us to construct a counterexample showing that for $p=\frac{n}{n+k}, k=0,1,2, \ldots, h_{r}^{p}(\Omega) \neq h_{z}^{p}(\Omega)$ (Theorem 2).

\section{2. $h^{p}$ Distributions SUPPORTED IN A HYPERPlane}

For $n \geq 2$, write $\mathbf{R}^{n}=\mathbf{R}^{n-1} \times \mathbf{R}$, and let $\Pi=\mathbf{R}^{n-1}=\left\{x_{n}=0\right\}$ be our chosen hyperplane. If $f$ is a distribution in $\mathbf{R}^{n}$ of order $K$ with compact support in $\Pi$, then

$$
f(\phi)=\sum_{k=0}^{K} f_{k}\left(\phi_{k}\right),
$$

where each $f_{k}$ is a distribution of compact support and order $K-k$ in $\mathbf{R}^{n-1}$, and

$$
\phi_{k}\left(x^{\prime}\right)=\left.\partial_{x_{n}}^{k} \phi\left(x^{\prime}, x_{n}\right)\right|_{x_{n}=0}
$$

(see [H], Theorem 2.3.5). Let $N$ be the largest integer such that $f_{N}$ is non-zero. We will call $N$ the order of $f$ in the normal direction. Then we have the following:

Theorem 1. A distribution $f$ in $\mathbf{R}^{n}$ with compact support in $\Pi$ is in the local Hardy space $h^{p}\left(\mathbf{R}^{n}\right), 0<p \leq 1$, only if its order $N$ in the normal direction satisfies

$$
N<n(1 / p-1) \text {. }
$$

Note that for $p=1$, this agrees with the fact that the only distribution supported in $\Pi$ which is in $h^{1}$ is the zero distribution, since elements of $h^{1}$ are functions.

To characterize the spaces $h^{p}\left(\mathbf{R}^{n}\right)$, we will use a "local grand maximal function" $m(f)$ defined using "normalized bump functions". Such a function $\varphi$ is smooth $\left(\mathcal{C}^{\infty}\right)$, supported in a ball of radius $r$, and satisfies

$$
\left|\partial^{\alpha} \varphi\right| \leq r^{-n-|\alpha|}
$$

for all multi-exponents $\alpha=\left(\alpha_{1}, \ldots, \alpha_{n}\right)$ with

$$
|\alpha|=\alpha_{1}+\ldots+\alpha_{n} \leq N_{p}+1
$$

where $N_{p}$ is the greatest integer in $n(1 / p-1)$.

For a tempered distribution $f$, we define

$$
m(f)(x)=\sup _{\varphi_{r}^{x}} f\left(\varphi_{r}^{x}\right)
$$

where the supremum is taken over all normalized bump functions $\varphi_{r}^{x}$ supported in balls of radii $r \leq 1$ containing $x$. Then $f \in h^{p}\left(\mathbf{R}^{n}\right)$ if and only if

$$
m(f) \in L^{p}\left(\mathbf{R}^{n}\right) .
$$

That this is equivalent to the definition given by Goldberg $([\mathrm{G}])$ can be seen from the remarks in $[\mathrm{S}], 5.17$.

In view of this characterization, the theorem will follow from the following

Lemma 1. Let $f$ be a distribution in $\mathbf{R}^{n}$ with compact support in $\Pi$, and let $N$ be the order of $f$ in the normal direction. Then for $0<p \leq 1$, the local grand maximal function $m(f)$ satisfies

$$
\int_{\mathbf{R}^{n-1}} m(f)^{p}\left(x^{\prime}, x_{n}\right) d x^{\prime} \geq C x_{n}{ }^{n-1-(n+N) p}
$$

for all sufficiently small $x_{n}>0$. 
Note that when $N \geq n(1 / p-1), n-1-(n+N) p \leq-1$ so the lemma shows that $m(f)^{p}$ is not locally integrable near $x_{n}=0$. This proves the theorem.

Proof of Lemma 1. Write

$$
f(\phi)=\sum_{k=0}^{N} f_{k}\left(\phi_{k}\right),
$$

as in equation 1, with $f_{N}$ nonzero. We may assume the support of $f$ lies in the interior of the unit cube $Q=[0,1]^{n-1}$. Since $f_{N}$ is nonzero, there is a smooth function $\phi$ such that $f_{N}(\phi)=\epsilon>0$. We may also take the support of $\phi$ to lie inside $Q$. By changing $\epsilon$ if necessary, we normalize $\phi$ so that

$$
\sup _{|\gamma| \leq N_{p}+1}\left|\partial_{x^{\prime}}^{\gamma} \phi\right| \leq 1
$$

Consider the dyadic cubes $Q_{\alpha}^{j}$, with $j=0,1,2, \ldots$ and $\alpha=\left(\alpha_{1}, \ldots, \alpha_{n-1}\right)$, $0 \leq \alpha_{i} \leq 2^{j}-1$, where the side length of $Q_{\alpha}^{j}$ is $2^{-j}$ and its closest corner to the origin lies at the point $\left(\alpha_{1} / 2^{j}, \ldots, \alpha_{n-1} / 2^{j}\right)$. Thus $Q=Q_{(0, \ldots, 0)}^{0}$.

For each $j$, we associate with the cubes $Q_{\alpha}^{j}$ a partition of unity $\left\{\eta_{\alpha}^{j}\right\}$, where each $\eta_{\alpha}^{j}$ is a smooth function supported in the double of the cube $Q_{\alpha}^{j}$, i.e. the cube $\widetilde{Q_{\alpha}^{j}}$ with the same center but double the side length, and such that

$$
\sum_{\alpha} \eta_{\alpha}^{j}=1
$$

on $Q$. Furthermore, we may assume that, for every $j$ and $\alpha$,

$$
\left|\partial_{x^{\prime}}^{\gamma}\left(\eta_{\alpha}^{j}\right)\right| \leq 2^{j|\gamma|}
$$

for all derivatives of order $|\gamma| \leq N_{p}+1$.

Set

$$
\phi_{\alpha}^{j}=A_{n, p} 2^{j(n-1)} \phi \eta_{\alpha}^{j},
$$

where the constant $A_{n, p}$ is chosen so that

$$
\left|\partial_{x^{\prime}}^{\gamma}\left(\phi_{\alpha}^{j}\right)\right| \leq 2^{j(n-1+|\gamma|)}
$$

for all $|\gamma| \leq N_{p}+1$. Consider a smooth one-variable function $\psi(t)$ with

$$
\psi(t)=\frac{1}{N !} t^{N}
$$

for $t \in[-1 / 2,1 / 2], \psi(t)=0$ for $|t|>1$, and $\left|d_{t}^{m} \psi(t)\right| \leq 1$ for $m \leq N_{p}+1$. Let

$$
\psi_{j}(t)=2^{j} \psi\left(2^{j} t\right)
$$

and

$$
\Phi_{\alpha}^{j}\left(x^{\prime}, x_{n}\right)=C_{n, p} \phi_{\alpha}^{j}\left(x^{\prime}\right) \psi_{j}\left(x_{n}\right),
$$

where again we chose the constant $C_{n, p}$ so that

$$
\left|\partial^{\beta}\left(\Phi_{\alpha}^{j}\right)\right| \leq\left(2^{-j} \sqrt{n}\right)^{-(n+|\beta|)}
$$

for all $|\beta| \leq N_{p}+1$. Since $\Phi_{\alpha}^{j}$ is supported in the cube $\widetilde{Q_{\alpha}^{j}} \times\left[-2^{-j}, 2^{-j}\right]$ of side length $2^{-j+1}$, hence in a ball of radius $2^{-j} \sqrt{n}$, this makes $\Phi_{\alpha}^{j}$ a normalized bump function.

Now note that

$$
f\left(\Phi_{\alpha}^{j}\right)=f_{N}\left(\left(\Phi_{\alpha}^{j}\right)_{N}\right)=C_{n, p^{2}}{ }^{j(N+1)} f_{N}\left(\phi_{\alpha}^{j}\right) .
$$


Thus for $x \in \widetilde{Q_{\alpha}^{j}} \times\left[-2^{-j}, 2^{-j}\right]$,

$$
m(f) \geq C_{n, p} 2^{j(N+1)}\left|f_{N}\left(\phi_{\alpha}^{j}\right)\right| .
$$

Recall that, for every $j$,

$$
\sum_{\alpha} f_{N}\left(\phi_{\alpha}^{j}\right)=A_{n, p} 2^{j(n-1)} f_{N}(\phi)=A_{n, p} 2^{j(n-1)} \epsilon .
$$

Therefore for $2^{-(j+1)} \leq x_{n} \leq 2^{-j}$,

$$
\begin{aligned}
\int_{\mathbf{R}^{n-1}} m(f)^{p}\left(x^{\prime}, x_{n}\right) d x^{\prime} & \geq C_{n, p} \sum_{\alpha}\left(2^{j(N+1)}\left|f_{N}\left(\phi_{\alpha}^{j}\right)\right|\right)^{p}\left|Q_{\alpha}^{j}\right| \\
& \geq C_{n, p} 2^{j(N+1) p} 2^{-j(n-1)}\left|\sum_{\alpha} f_{N}\left(\phi_{\alpha}^{j}\right)\right|^{p} \\
& =C_{n, p}^{\prime} 2^{j(N+1) p} 2^{-j(n-1)} 2^{j(n-1) p} \epsilon^{p} \\
& =C_{n, p}^{\prime} \epsilon^{p} 2^{-j[n-1-(n+N) p]} \\
& \geq C_{n, p, N, \epsilon} x_{n}^{n-1-(n+N) p} .
\end{aligned}
$$

This proves the lemma.

We will now give an example to show that Theorem 1 does not hold with $N$ replaced by $K$, the total order of the distribution.

Example 1. For $0<p<1$, there exists a distribution $f \in h^{p}\left(\mathbf{R}^{n}\right)$ with compact support contained in a hyperplane, such that the order $K$ of $f$ satisfies

$$
K \geq N_{p}
$$

where $N_{p}$ is the greatest integer in $n(1 / p-1)$.

Take the hyperplane $\Pi=\mathbf{R}^{n-1}$ as above. In $\mathbf{R}^{n-1}$, consider cubes $Q_{j}$ with centers $c_{j}$ and side lengths $\delta_{j}$, such that the double cubes $2 Q_{j}$ are pairwise disjoint and $\sum \delta_{j}<\infty$. Take functions $a_{j}$ of the $n-1$ variables $x^{\prime}=x_{1}, \ldots, x_{n-1}$ (respectively) supported in $Q_{j}$ and satisfying

$$
\begin{gathered}
\left\|a_{j}\right\|_{\infty} \leq \delta_{j}{ }^{1-n / p}, \\
\int a_{j}\left(x^{\prime}\right)\left(x^{\prime}\right)^{\alpha} d x^{\prime}=0
\end{gathered}
$$

for all $\alpha=\left(\alpha_{1}, \ldots, \alpha_{n-1}\right)$ with $|\alpha| \leq N_{p}$, and

$$
\int a_{j}\left(x^{\prime}\right)\left(x_{1}-\left(c_{j}\right)_{1}\right)^{N_{p}+1} d x^{\prime}=C_{n} \delta_{j}^{n-n / p+N_{p}+1}
$$

for some $C_{n}>0$.

Define linear functionals $\tau_{j}$ on $\mathcal{S}\left(\mathbf{R}^{n}\right)$ by

$$
\tau_{j}(\varphi)=\int_{\mathbf{R}^{n-1}} a_{j}\left(x^{\prime}\right) \varphi\left(x^{\prime}, 0\right) d x^{\prime}
$$

for every $\varphi \in \mathcal{S}\left(\mathbf{R}^{n}\right)$. By expanding $\varphi\left(x^{\prime}, 0\right)$ in a Taylor expansion around $c_{j}$, one can see that

$$
\left|\tau_{j}(\varphi)\right| \leq C\|\varphi\|_{\mathcal{C}^{N_{p}+1}} \delta_{j}{ }^{n-n / p+N_{p}+1} \leq C_{n, p}\|\varphi\|_{\mathcal{C}^{N_{p}+1}}
$$


since $\delta_{j} \rightarrow 0$ and $n-n / p+N_{p}+1>0$. This shows the $\tau_{j}$ are continuous on $\mathcal{S}\left(\mathbf{R}^{n}\right)$, and we can define $f \in \mathcal{S}^{\prime}\left(\mathbf{R}^{n}\right)$ by

$$
f=\sum \lambda_{j} \tau_{j}
$$

where the sequence $\lambda_{j}$ is chosen to satisfy $\sum \lambda_{j}{ }^{p}<\infty$ and $\lambda_{j} / \delta_{j} \rightarrow \infty$ as $j \rightarrow \infty$ (for example take $\delta_{j}=2^{-j}, \lambda_{j}=j^{-1-1 / p}$ ).

To see that the order of $f$ is at least $N_{p}$, take a sequence of smooth functions $\varphi_{j} \in \mathcal{S}\left(\mathbf{R}^{n}\right)$ with $\varphi_{j}\left(x^{\prime}, 0\right)$ supported in $2 Q_{j}$,

$$
\left|\partial^{\alpha} \varphi_{j}\right| \leq 1
$$

for $|\alpha| \leq N_{p}-1$, and

$$
\varphi_{j}\left(x^{\prime}, 0\right)=\frac{1}{\left(N_{p}+1\right) !}\left(x_{1}-\left(c_{j}\right)_{1}\right)^{N_{p}+1} \delta_{j}^{-2}
$$

on $Q_{j}$. Thus

$$
f\left(\varphi_{j}\right)=\lambda_{j} \int_{Q_{j}} a_{j}\left(x^{\prime}\right) \varphi_{j}\left(x^{\prime}, 0\right) d x^{\prime}=C_{n, p} \lambda_{j} \delta_{j}^{n-n / p+N_{p}+1-2} \rightarrow \infty
$$

as $j \rightarrow \infty$, since $n-n / p+N_{p}+1-2 \leq-1$.

Finally, to see that $f \in h^{p}\left(\mathbf{R}^{n}\right)$, consider the local grand maximal function $m(f)$. It suffices to show $\left\|m\left(\tau_{j}\right)\right\|_{L^{p}\left(\mathbf{R}^{n}\right)} \leq C$ uniformly in $j$. Take $x \in \mathbf{R}^{n}$. Note that if $\varphi_{t}^{x}$ is a normalized bump function supported in a ball of radius $t$ containing $x$, then $\tau_{j}\left(\varphi_{t}^{x}\right) \neq 0$ only if $t \geq x_{n} / 2$, and $t \varphi_{t}^{x}\left(y^{\prime}, 0\right)$ is a normalized bump function in $n-1$ variables. Thus

$$
m\left(\tau_{j}\right)(x) \leq \frac{2}{x_{n}} m_{\mathbf{R}^{n-1}}\left(a_{j}\right)\left(x^{\prime}\right),
$$

where $m_{\mathbf{R}^{n-1}}$ denotes the local grand maximal function on $\mathbf{R}^{n-1}$. Recalling that $m_{\mathbf{R}^{n-1}}$ is bounded on $L^{2}\left(\mathbf{R}^{n-1}\right)$, we get that

$$
\begin{aligned}
\int_{2 Q_{j} \times\left[-\delta_{j}, \delta_{j}\right]} m\left(\tau_{j}\right)^{p}(x) d x & \leq C \int_{-\delta_{j}}^{\delta_{j}} x_{n}{ }^{-p} \int_{2 Q_{j}} m_{\mathbf{R}^{n-1}}\left(a_{j}\right)^{p}\left(x^{\prime}\right) d x^{\prime} \\
& \leq C \delta_{j}^{1-p}\left\|m\left(a_{j}\right)\right\|_{L^{2}\left(\mathbf{R}^{n-1}\right)}^{p}\left|2 Q_{j}\right|^{1-p / 2} \\
& \leq C \delta_{j}^{1-p}\left\|a_{j}\right\|_{L^{2}\left(\mathbf{R}^{n-1}\right)}^{p}\left|2 Q_{j}\right|^{1-p / 2} \\
& \leq C \delta_{j}{ }^{1-p} \delta_{j}{ }^{p-n}\left|Q_{j}\right| \\
& =C .
\end{aligned}
$$

Note that here we used the fact that $p<1$.

For $x \notin 2 Q_{j} \times\left[-\delta_{j}, \delta_{j}\right]$, if $\varphi_{t}^{x}$ is a normalized bump function supported in a ball of radius $t$ containing $x$, then as above

$$
\begin{aligned}
\left|\tau_{j}\left(\varphi_{t}^{x}\right)\right| & \leq C\left\|\varphi_{t}^{x}\right\|_{\mathcal{C}^{N_{p}+1}} \delta_{j}{ }^{n-n / p+N_{p}+1} \\
& \leq C t^{-n-\left(N_{p}+1\right)} \delta_{j}^{n-n / p+N_{p}+1} \\
& \leq C\left|x-\left(c_{j}, 0\right)\right|^{-n-\left(N_{p}+1\right)} \delta_{j}{ }^{n-n / p+N_{p}+1}
\end{aligned}
$$


so

$$
\begin{aligned}
& \int_{x \notin 2 Q_{j} \times\left[-\delta_{j}, \delta_{j}\right]} m\left(\tau_{j}\right)^{p}(x) d x \\
& \quad \leq C \delta_{j}^{n p-n+\left(N_{p}+1\right) p} \int_{\left|x-\left(c_{j}, 0\right)\right| \geq \delta_{j}}\left|x-\left(c_{j}, 0\right)\right|^{-n p-\left(N_{p}+1\right) p} d x \\
& \quad=C .
\end{aligned}
$$

This completes the example.

$$
\text { 3. THE SPACES } h_{r}^{p}(\Omega) \text { AND } h_{z}^{p}(\Omega)
$$

Let $\Omega$ be a bounded domain in $\mathbf{R}^{n}$, with smooth boundary. Let us recall the definitions of the spaces $h_{r}^{p}(\Omega)$ and $h_{z}^{p}(\Omega)$, given in [CKS]:

$$
h_{r}^{p}(\Omega)=\left\{f \in \mathcal{D}^{\prime}(\Omega): \exists F \in h^{p}\left(\mathbf{R}^{n}\right),\left.F\right|_{\Omega}=f\right\}
$$

and

$$
h_{z}^{p}(\Omega)=\left\{f \in \mathcal{D}^{\prime}(\Omega): \exists F \in h^{p}\left(\mathbf{R}^{n}\right),\left.F\right|_{\Omega}=f,\left.F\right|_{\mathbf{R}^{n} \backslash \bar{\Omega}}=0\right\} .
$$

Clearly $h_{z}^{p}(\Omega)$ is a subspace of $h_{r}^{p}(\Omega)$. It was shown in [CDS] that in fact $h_{r}^{p}(\Omega)=$ $h_{z}^{p}(\Omega)$ for all values of $p$ with $\frac{n}{n+j+1}<p<\frac{n}{n+j}, j=0,1, \ldots$. We will now show that this is not true when $n(1 / p-1)$ is an integer.

Theorem 2. For $p=1, \frac{n}{n+1}, \frac{n}{n+2}, \ldots$,

$$
h_{r}^{p}(\Omega) \neq h_{z}^{p}(\Omega)
$$

Proof. Assume $0 \in \partial \Omega$, and let $U \subset V$ be open neighborhoods of 0 . We may take these so that there is a smooth choice of coordinates in $V$ in which $V=B(0,2)$, $U=B(0,1)$ and $\Omega \cap V=\mathbf{R}_{+}^{n} \cap B(0,2)$. Here $\mathbf{R}_{+}^{n}$ denotes the open upper half-space $\left\{x: x_{n}>0\right\}$.

We will construct a distribution $f \in h_{r}^{p}(\Omega)$, supported in $U$, such that there is no distribution $G \in h^{p}\left(\mathbf{R}^{n}\right)$ satisfying

$$
\left.G\right|_{\Omega}=f
$$

and

$$
\left.G\right|_{\mathbf{R}^{n} \backslash \bar{\Omega}}=0
$$

Note that the space of distributions in $h_{r}^{p}(\Omega)$ which are supported in $U$ does not depend on the choice of coordinates.

Let us construct $f$ as follows. For $j=1,2, \ldots$, consider the cylinder

$$
S_{j}=B^{n-1}\left(0,2^{-(j+1)}\right) \times\left[2^{-(j+1)}, 2^{-j}\right]
$$

where $B^{n-1}(0, r)$ denotes the $(n-1)$-dimensional ball of radius $r$ in the variables $x^{\prime}=x_{1}, \ldots, x_{n-1}$. Note that $S_{j}$ is contained in the (much larger) cube

$$
Q_{j}=\left[-2^{-(j+1)}, 2^{-(j+1)}\right]^{n-1} \times\left[2^{-(j+1)}, 3 \cdot 2^{-(j+1)}\right]
$$

of volume $\left|Q_{j}\right|=2^{-j n}$. Set

$$
a_{j}=\left|Q_{j}\right|^{-1 / p} \chi_{S_{j}}
$$

If $\lambda_{j}=j^{-(1+1 / p)}$, then $\sum \lambda_{j}{ }^{p}<\infty$ so by the atomic decomposition for $h_{r}^{p}$ (see [CKS], Theorem 2.7,) the distribution $f \in \mathcal{D}^{\prime}(\Omega)$ defined by

$$
f=\sum_{j=1}^{\infty} \lambda_{j} a_{j}
$$


is an element of $h_{r}^{p}(\Omega)$. Furthermore, $f$ is supported in $\Gamma \cap B(0,1)$, where $\Gamma$ is the cone

$$
\left\{x \in \mathbf{R}^{n}:\left|x^{\prime}\right| \leq x_{n}<1\right\} .
$$

In order to extend $f$ to a distribution $F \in \mathcal{S}^{\prime}\left(\mathbf{R}^{n}\right)$, we define distributions $\tau_{j}$ by

$$
\tau_{j}(\varphi)=\int a_{j}(x)\left[\varphi(x)-\sum_{|\alpha| \leq N_{p}-1} \frac{1}{\alpha !} \partial^{\alpha} \varphi(0) x^{\alpha}\right] d x
$$

for every $\varphi \in \mathcal{S}\left(\mathbf{R}^{n}\right)$. Since

$$
\begin{aligned}
\left|\tau_{j}(\varphi)\right| & =\left|\int a_{j}(x)\left[\varphi(x)-\sum_{|\alpha| \leq N_{p}-1} \frac{1}{\alpha !} \partial^{\alpha} \varphi(0) x^{\alpha}\right] d x\right| \\
& \leq C 2^{j n / p}\|\varphi\|_{\mathcal{C}^{N_{p}}} \int_{Q_{j}}|x|^{N_{p}} d x \\
& \leq C 2^{j\left(n / p-N_{p}-n\right)}\|\varphi\|_{\mathcal{C}^{N_{p}}} \\
& =C\|\varphi\|_{\mathcal{C}^{N_{p}}}
\end{aligned}
$$

and $\sum \lambda_{j}<\infty$, we can define a distribution $F \in \mathcal{S}^{\prime}\left(\mathbf{R}^{n}\right)$ by

$$
F=\sum_{j=1}^{\infty} \lambda_{j} \tau_{j}
$$

This distribution is supported in $B(0,1) \cap \bar{\Omega}$ and satisfies

$$
\left.F\right|_{\Omega}=\left.\sum_{j=1}^{\infty} \lambda_{j} \tau_{j}\right|_{\Omega}=\sum_{j=1}^{\infty} \lambda_{j} a_{j}=f
$$

Now suppose there was a distribution $G \in \mathcal{S}^{\prime}\left(\mathbf{R}^{n}\right)$ with $G$ supported on $\bar{\Omega}$ and $\left.G\right|_{\Omega}=f$. We want to show that $G$ cannot be an element of $h^{p}\left(\mathbf{R}^{n}\right)$. Since $h^{p}\left(\mathbf{R}^{n}\right)$ is closed under multiplication by smooth functions of compact support, we may assume $G$ is supported in $V=B(0,2)$.

Consider the distribution $G-F \in \mathcal{S}^{\prime}\left(\mathbf{R}^{n}\right)$. Since both $G$ and $F$ are supported on $\bar{\Omega}$, and $\left.G\right|_{\Omega}=f=\left.F\right|_{\Omega}$, we must have that $G-F$ is supported on $\partial \Omega$, and in particular on $B^{n-1}(0,2)=B(0,2) \cap \partial \mathbf{R}_{+}^{n}$. Thus $G-F$ is of the form of equation (1).

Let $N$ denote the order of $G-F$ in the normal direction.

Claim 1. If $N<N_{p}=n(1 / p-1)$, then the distribution $G$ does not belong to $h^{p}\left(\mathbf{R}^{n}\right)$, since its local grand maximal function satisfies

$$
m(G)(x) \geq C|x|^{-n / p}\left(\log \frac{1}{|x|}\right)^{-1 / p}
$$

for $x \in \Gamma, x \neq 0$.

To get the lower bound for $m(G)$, we will construct a special test function $\phi$. First take a smooth function $\psi$ of one variable, supported in $(-1,2)$, with $\psi(y) \geq 0$ for $y \geq 0$,

and

$$
\psi(y)=\frac{y^{N_{p}}}{N_{p} !} \text { for } y \in[0,1]
$$

$$
\left|\frac{d^{k}}{d y^{k}}(\psi)\right| \leq 1
$$


for $k \leq N_{p}+1$. Now take $\varphi$ to be a smooth function of $n-1$ variables, supported in $B^{n-1}(0,2)$, with $\varphi \geq 0, \varphi=1$ on $B^{n-1}(0,1)$ and

$$
\left|\partial^{\alpha}(\varphi)\right| \leq 1
$$

for $|\alpha| \leq N_{p}+1$. Defining $\phi$ by

$$
\phi(x)=\varphi\left(x^{\prime}\right) \psi\left(x_{n}\right),
$$

we see that $\phi$ is supported in $B(0,3)$,

$$
\phi(x)=\frac{x_{n}{ }^{N_{p}}}{N_{p} !}
$$

for $x \in B^{n-1}(0,1) \times[0,1]$, and

$$
\left|\partial^{\alpha}(\phi)\right| \leq 1
$$

for $|\alpha| \leq N_{p}+1$.

Suppose $x \in \Gamma, x \neq 0$. Let $x^{*}=\left(x^{\prime}, 0\right)$, and define

$$
\phi_{x}(y)=3^{-n-N_{p}}|x|^{-n} \phi\left(\frac{y-x^{*}}{|x|}\right) .
$$

Then $\phi_{x}$ is supported in the ball $B\left(x^{*}, 3|x|\right)$, with $\phi_{x} \geq 0$ on $\mathbf{R}_{+}^{n}$ and

$$
\phi_{x}(y)=\frac{y_{n}{ }^{N_{p}}}{N_{p} !(3|x|)^{n+N_{p}}}
$$

for $y \in S_{x}=B^{n-1}\left(x^{\prime},|x|\right) \times[0,|x|]$. Furthermore

$$
\left|\partial^{\alpha}\left(\phi_{x}\right)\right| \leq(3|x|)^{-n-|\alpha|}
$$

for $|\alpha| \leq N_{p}+1$. Thus $\phi_{x}$ is a normalized bump function.

Note that, for $k<N_{p}$,

$$
\left.\frac{\partial^{k}}{\partial y_{n}{ }^{k}} \phi_{x}\left(y^{\prime}, y_{n}\right)\right|_{y_{n}=0}=3^{-n-N_{p}}|x|^{-n-k} \varphi\left(\frac{y^{\prime}-x^{\prime}}{|x|}\right) \frac{\partial^{k} \psi}{\partial y_{n}{ }^{k}}(0)=0
$$

so by equation (1), if $N<N_{p}$,

$$
(G-F)\left(\phi_{x}\right)=0 .
$$

Moreover, $\tau_{j}\left(\phi_{x}\right)=\int a_{j} \phi_{x}$ for all $j$. Thus

$$
\begin{aligned}
G\left(\phi_{x}\right) & =F\left(\phi_{x}\right) \\
& \geq \sum_{S_{j} \subset S_{x}} \lambda_{j} \int_{S_{j}} a_{j}(y) \phi_{x}(y) d y \\
& =\frac{1}{N_{p} !(3|x|)^{n+N_{p}}} \sum_{S_{j} \subset S_{x}} \lambda_{j} \int_{S_{j}} a_{j}(y) y_{n}{ }^{N_{p}} d y \\
& =C_{N_{p}}(3|x|)^{-n-N_{p}} \sum_{S_{j} \subset S_{x}} \lambda_{j} 2^{j\left(n / p-N_{p}-n\right)} \\
& \geq C_{N_{p}}^{\prime}|x|^{-n-N_{p}} \sum_{j \geq-\log _{2} x_{n}+2} j^{-1 / p-1} \\
& \approx|x|^{-n / p}\left(\log \frac{1}{|x|}\right)^{-1 / p} .
\end{aligned}
$$


Here we have used the fact that for $x \in \Gamma$, if $2^{-j} \leq(\sqrt{2}-1) x_{n}$, then

$$
S_{j}=B^{n-1}\left(0,2^{-(j+1)}\right) \times\left[2^{-(j+1)}, 2^{-j}\right] \subset S_{x}=B^{n-1}\left(x^{\prime},|x|\right) \times[0,|x|] .
$$

Claim 2. If $N \geq N_{p}$, then the distribution $G$ does not belong to $h^{p}\left(\mathbf{R}^{n}\right)$, since its local grand maximal function satisfies

$$
\int_{\mathbf{R}^{n-1}} m(G)^{p}\left(x^{\prime}, x_{n}\right) d x^{\prime} \geq C x_{n}^{-1}
$$

for all sufficiently small $x_{n}>0$.

By Lemma 1, with $N \geq N_{p}$, the distribution $G-F$ satisfies

$$
\int_{\mathbf{R}^{n-1}} m(G-F)^{p}\left(x^{\prime}, x_{n}\right) d x^{\prime} \geq C x_{n}{ }^{n-1-\left(n+N_{p}\right) p}=C x_{n}{ }^{-1}
$$

for all sufficiently small $x_{n}>0$. Since

$$
\int_{\mathbf{R}^{n-1}} m(G)^{p}\left(x^{\prime}, x_{n}\right) d x^{\prime} \geq \int_{\mathbf{R}^{n-1}} m(G-F)^{p}\left(x^{\prime}, x_{n}\right) d x^{\prime}-\int_{\mathbf{R}^{n-1}} m(F)^{p}\left(x^{\prime}, x_{n}\right) d x^{\prime},
$$

it suffices to prove that

$$
\int_{\mathbf{R}^{n-1}} m(F)^{p}\left(x^{\prime}, x_{n}\right) d x^{\prime}=o\left(x_{n}{ }^{-1}\right)
$$

as $x_{n} \rightarrow 0$. In fact, we will show that

$$
\int_{\mathbf{R}^{n-1}} m(F)^{p}\left(x^{\prime}, x_{n}\right) d x^{\prime} \leq C\left(x_{n} \log \left(1 / x_{n}\right)\right)^{-1}
$$

for sufficiently small $x_{n}>0$.

To prove the upper bound on the maximal function of $F$, take $x \in \mathbf{R}_{+}^{n}$, and let $\varphi_{t}^{x}$ be a bump function supported in a ball of radius $t \leq 1$ containing $x$. Since $F$ is supported in $B(0,1)$, we can assume $|x|<3$.

If $t \geq|x| / 4$, write

$$
F\left(\varphi_{t}^{x}\right)=\sum_{j=1}^{\infty} \lambda_{j} \tau_{j}\left(\varphi_{t}^{x}\right) .
$$

Note that if $j<-\log _{2}(t)-4$, then $2^{-(j+1)}>8 t>2 t+x_{n}$, so $\varphi_{t}^{x}=0$ on $S_{j}$ and

$$
\begin{aligned}
\sum_{j<-\log _{2}(t)-4}\left|\lambda_{j} \tau_{j}\left(\varphi_{t}^{x}\right)\right| & =\sum_{j<-\log _{2}(t)-4}\left|\lambda_{j} 2^{j n / p} \sum_{|\alpha| \leq N_{p}-1} \frac{1}{\alpha !} \partial^{\alpha} \varphi_{t}^{x}(0) \int_{S_{j}} x^{\alpha} d x\right| \\
& \leq C \sum_{j<-\log _{2}(t)-4} \lambda_{j} \sum_{|\alpha| \leq N_{p}-1} \frac{1}{\alpha !} t^{-n-|\alpha|} 2^{j(n / p-n-|\alpha|)} \\
& \leq C \sum_{|\alpha| \leq N_{p}-1} \frac{1}{\alpha !} t^{-n-|\alpha|} \sum_{j<-\log _{2}(t)-4} \frac{2^{j(n / p-n-|\alpha|)}}{j^{1+1 / p}} \\
& \leq C \sum_{|\alpha| \leq N_{p}-1} \frac{1}{\alpha !} t^{-n-|\alpha|} \frac{t^{-(n / p-n-|\alpha|)}}{\log (1 / t)^{1 / p}} \\
& \leq C t^{-n / p}(\log (1 / t))^{-1 / p}
\end{aligned}
$$


On the other hand,

$$
\begin{aligned}
\sum_{j \geq-\log _{2}(t)-4}\left|\lambda_{j} \tau_{j}\left(\varphi_{t}^{x}\right)\right| & \leq C \sum_{j \geq-\log _{2}(t)-4} \frac{1}{j^{1+1 / p}}\left\|\varphi_{t}^{x}\right\|_{\mathcal{C}^{N_{p}}} \\
& \leq C t^{-n-N_{p}} \sum_{j \geq-\log _{2}(t)-4} \frac{1}{j^{1+1 / p}} \\
& \leq C t^{-n / p}(\log (1 / t))^{-1 / p}
\end{aligned}
$$

Thus

$$
\begin{aligned}
\left|F\left(\varphi_{t}^{x}\right)\right| & \leq C t^{-n / p}(\log (1 / t))^{-1 / p} \\
& \leq C|x|^{-n / p}(\log (1 /|x|))^{-1 / p}
\end{aligned}
$$

since $(|x| / t)^{n / p}(\log |x| / \log t)^{1 / p}$ remains bounded when $|x|<<t$.

If $t<|x| / 4$, then $\varphi_{t}^{x}$ vanishes in a neighborhood of 0 , and $S_{j} \cap \operatorname{supp}\left(\varphi_{t}^{x}\right) \neq \emptyset$ only if $j \leq 3 / 2-\log _{2}|x|$, so

$$
\begin{aligned}
\left|F\left(\varphi_{t}^{x}\right)\right| & \leq C \sum_{j \leq-C \log |x|} \lambda_{j}\left|\int a_{j} \varphi_{t}^{x}\right| \\
& \leq C \sum_{j \leq-C \log |x|} \frac{1}{j^{1+1 / p}} 2^{j n / p}\left\|\varphi_{t}^{x}\right\|_{L^{1}} \\
& \leq C|x|^{-n / p}(\log (1 /|x|))^{-1 / p}
\end{aligned}
$$

In both cases we are assuming of course that $|x|$ is sufficiently small, i.e. $|x| \leq$ $a<<1$, so that $\log (1 /|x|)$ is bounded below. Otherwise we just have

$$
\left|F\left(\varphi_{t}^{x}\right)\right| \leq C|x|^{-n / p} \sum \lambda_{j} \leq C_{a} .
$$

Now integrating in the first $n-1$ variables, we have

$$
\begin{array}{rl}
\int_{\mathbf{R}^{n-1}} & m(F)^{p}\left(x^{\prime}, x_{n}\right) d x^{\prime}= \\
& \int_{\left|x^{\prime}\right| \leq x_{n}} m(F)^{p}\left(x^{\prime}, x_{n}\right) d x^{\prime}+\int_{x_{n} \leq\left|x^{\prime}\right| \leq a} m(F)^{p}\left(x^{\prime}, x_{n}\right) d x^{\prime} \\
& +\int_{a \leq\left|x^{\prime}\right| \leq 3} m(F)^{p}\left(x^{\prime}, x_{n}\right) d x^{\prime} \\
\leq & C \int_{\left|x^{\prime}\right| \leq x_{n}} x_{n}^{-n}\left(\log \left(1 / x_{n}\right)\right)^{-1} d x^{\prime} \\
& +C \int_{x_{n} \leq\left|x^{\prime}\right| \leq a}\left|x^{\prime}\right|^{-n}\left(\log \left(1 /\left|x^{\prime}\right|\right)\right)^{-1} d x^{\prime}+C \\
\leq & C x_{n}^{-1}\left(\log \left(1 / x_{n}\right)\right)^{-1}+C \int_{x_{n}}^{a} \frac{d r}{r^{2}(-\log r)}+C .
\end{array}
$$

But

$$
\begin{aligned}
\int_{x_{n}}^{a} \frac{d r}{r^{2}(-\log r)} & =\int_{x_{n}}^{a} \frac{d r}{r^{2} \log ^{2} r}+x_{n}^{-1}\left(\log \frac{1}{x_{n}}\right)^{-1}+C \\
& \leq(-\log a)^{-1} \int_{x_{n}}^{a} \frac{d r}{r^{2}(-\log r)}+x_{n}^{-1}\left(\log \left(1 / x_{n}\right)\right)^{-1}+C
\end{aligned}
$$


SO

$$
\int_{x_{n}}^{a} \frac{d r}{r^{2}(-\log r)} \leq C x_{n}{ }^{-1}\left(\log \left(1 / x_{n}\right)\right)^{-1}+C
$$

for $a$ sufficiently small.

This concludes the proof of Theorem 2 .

\section{REFERENCES}

[CDS] D.C. Chang, G. Dafni, and E. M. Stein, Hardy spaces, BMO, and boundary value problems for the Laplacian on a smooth domain in $\mathbf{R}^{n}$, Trans. Amer. Math. Soc., to appear. CMP 97:15

[CKS] D.C. Chang, S. G. Krantz, and E. M. Stein, $H^{p}$ Theory on a smooth domain in $\mathbf{R}^{N}$ and elliptic boundary value problems, J. Funct. Anal. 114, No. 2 (1993), 286-347. MR 94j: 46032

[G] D. Goldberg, A local version of real Hardy spaces, Duke Math. J. 46 (1979), 27-42. MR 80h:46052

[H] L. Hörmander, The Analysis of Linear Partial Differential Operators I, Springer-Verlag, Berlin 1983. MR 85g:35002a

[JSW] A. Jonsson, P. Sjögren, and H. Wallin, Hardy and Lipschitz spaces on subsets of $\mathbf{R}^{n}$, Studia Math. 80, No. 2 (1984), 141-166. MR 87b:46022

[M] A. Miyachi, $H^{p}$ spaces over open subsets of $\mathbf{R}^{n}$, Studia Math. 95, No. 3 (1990), 205-228. MR 91m: 42022

[S] E. M. Stein, Harmonic Analysis: Real-Variable Methods, Orthogonality, and Oscillatory Integrals, Princeton Univ. Press, Princeton, New Jersey, 1993. MR 95c:42002

Department of Mathematics, Northwestern University, Evanston, Illinois 60208-2730

E-mail address: dafni@math.nwu.edu 